\title{
Fibers based on propagating bound states in the continuum
}

\author{
Evgeny N. Bulgakov ${ }^{1,2}$ ANd Almas F. SAdReev ${ }^{1, *}$ \\ 11 Kirensky Institute of Physics, Federal Research Center KSC SB RAS, 660036 Krasnoyarsk, Russia \\ ${ }^{22}$ Siberian State Aerospace University, Krasnoyarsk 660014, Russia \\ *Corresponding author: almas@tnp.krasn.ru
}

Compiled April 19, 2018

\begin{abstract}
We show that a circular periodic array of $N$ dielectric cylinders supports nearly bound states in the continuum (BICs) propagating along the cylinders. These propagating nearly BICs with extremely large $Q$ factors of order $\exp (\lambda N)$ are surrounded by resonant modes weakly leaking into the radiation continuum. We present leaky zones in the vicinity of different types of BICs: symmetry protected nearly BICs with the resonant width proportional to the squared propagation constant $\Gamma \sim k_{z}^{2}$, non-symmetry protected nearly BICs with finite propagation constant $k_{c}$ with $\Gamma \sim\left(k_{z}-k_{c}\right)^{2}$ and non-symmetry protected nearly BICs with $\Gamma \sim k_{z}^{4}$. The latter propagating nearly BICs can serve for transmission of electromagnetic signal paving a way to novel type of optical fibers. We also demonstrate weakly leaking resonant modes which carry orbital angular momentum.
\end{abstract}

(C) 2018 Optical Society of America under the terms of the OSA Open Access Publishing Agreement OCIS codes: (060.2420) Fibers, polarization-maintaining; (060.3735) Fiber Bragg gratings; (230.7400)Waveguides.

http://dx.doi.org/10.1364/optica.XX.XXXXXX

\section{INTRODUCTION}

Standard optical fibers guide light using total internal reflection. This restricts their optical properties, because only solid or liquid materials can be used for the fiber core. There are no suitable cladding materials which have a sufficiently low refractive index to confine light by total internal reflection in a vacuum or a gas core.

Substantial efforts have been invested over the past years in fabricating photonic crystals materials that have a periodic modulation of the refractive index on the scale of the optical wavelength. The interest in such materials lies in their ability to strongly reflect light of certain frequencies. For example, structure consisted of periodically designed layers forms onedimensional photonic crystal (PhC) which exhibit band gaps at optical frequencies (photonic band gaps) [1]. Light that is incident upon a band-gap material from the outside would be totally reflected. Similarly, light that existed at a structural-defect site in such a material would be permanently trapped, being unable to propagate through the lattice. Photonic band gap (PBG) structures offer the opportunity to design new optical properties into existing materials by wavelength-scale periodic micro structuring of the material morphology [2]. One can imagine that such a structure of order of ten layers can be rolled up to form cladding capable to almost perfectly trap light inside realizing fiber. Another design of two-dimensionally periodic structures in the form of long, fine silica fibers that have a regular array of tiny air holes running down their length constitute artificial two-dimensional $\mathrm{PhC}$ with lattice constants on the order of micrometers [3].

However, the demand of perfectness of such fibers enormously enlarges their cross-section. In the present paper we propose a different design of fibers based on the capability of a periodic array of dielectric cylinders to trap light at certain frequencies. The property is based on a fundamental family of localized solutions of Maxwell's equations, so called bound states in the continuum (BICs). Recently BICs with zero Bloch vector were reported in infinitely long periodic arrays of dielectric cylinders [4-20]. BICs propagating along the array were also shown to exist [20-28]. Our primary interest is in the BICs which can propagate along the cylinders $[25,28]$ giving rise to a new family of guided modes with frequencies above the light line. 


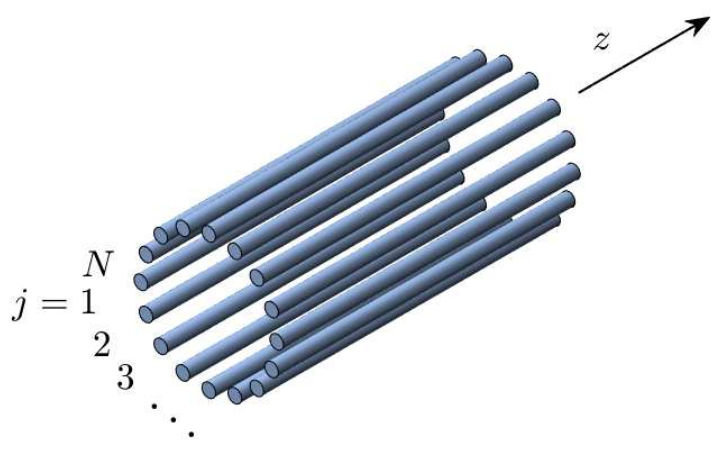

Fig. 1. $N$ infinitely long circular dielectric cylinders with radius $a$ stacked parallel to each other in a circle of radius $R$.

Physically, the occurrence of BICs in the infinite array of cylinders is the result of the periodicity of the array that quantizes the radiation continua in the form of diffraction continua $[17,20]$. Obviously, the infinite array of dielectric cylinders is an unrealistic limit. In practice we deal with finite number $N$ of cylinders which have material losses given by the imaginary part of refractive index, structural fluctuations of cylinders, the effect of substrate etc, transforming the ideal BIC into a resonant mode with small resonant width [13, 16, 17, 29, 30]. Although the full range study of these factors is still far from completion it was shown that the $Q$ factor of the symmetry protected quasi BICs grows quadratically with $N$.

However if the array of cylinders is rolled into a circle as shown in Fig. 1 the $Q$ factor grows exponentially with $N[31,32]$. In practice such Q-factors make the nearly BICs in the circular array indistinguishable from true BICs in the infinite array of cylinders [32].

In the present paper we demonstrate a few examples of the nearly BICs surrounded by propagating resonant modes weakly leaking into the radiation continuum. The property of the nearly BICs to serve as modes guided above the light line paves a way to new designs of fibers composed of $N$ dielectric cylinders circularly packed parallel to each other. These nearly BICs fill the core of the fiber and can carry orbital angular momentum (OAM) $m$. Each type of the above listed nearly BICs is hosted by a leaky zone with high $Q$ surrounding the nearly BIC.

\section{NEAR BICS PROPAGATING ALONG THE FIBER}

Here for brevity we omit the details of calculations. The calculations are based on the theory of scattering by a finite cluster of cylinders $[32,33]$. We start with the simplest symmetry protected standing wave nearly BIC whose coupling with the radiation continuum is exponentially weakened because of symmetry incompatibility [31,32]. This nearly BIC as shown in Fig. 2 originates from a true standing wave BIC in the infinite periodic array of dielectric cylinders at the $\Gamma$-point first reported by Shipman and Venakides [4]. The electric field of the BIC solu-

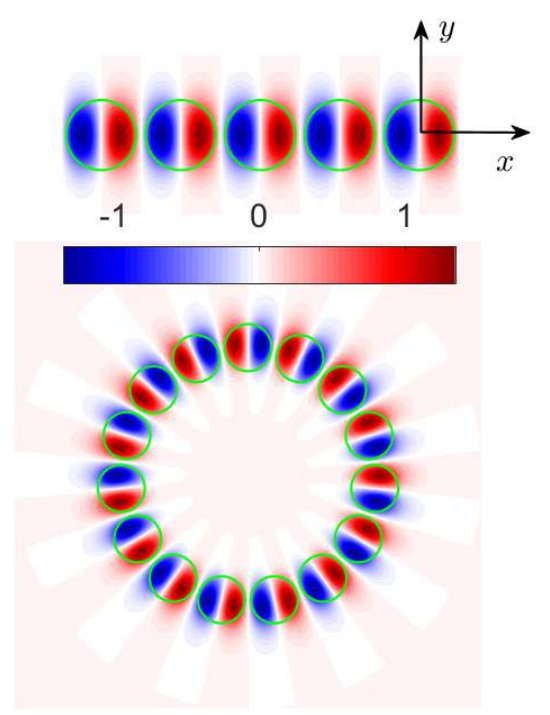

Fig. 2. z-component of the electric field of the symmetry protected standing wave BIC in the linear periodic array of dielectric cylinders with $a=0.44$ and $\epsilon=15$ with frequency $k_{0 c}=1.8315$ and its counterpart, nearly standing BIC in the circular array of the radius $R=7.25 a$ of 15 cylinders with frequency $k_{0 c}=1.837$ with $m=0$ from Ref [32]. The radius of the cylinders $a$ is given in terms of the azimuthal period of circular array $h=2 \pi R / N$ where $R$ is the radius of circular array. Respectively the wave numbers are given in terms of inverse $h$.

tion directed along the cylinders is even relative to the direction perpendicular to the plane of the array (y-axis in Fig. 2) and odd relative to $x \rightarrow-x$ where $x$ and $y$ are local coordinate system tied to the center of cylinder. The $\mathrm{z}$-component of magnetic field of the symmetry protected BIC equals zero to define the nearly BIC as E-polarized. When $N$ cylinders are rolled up into a circle still the symmetry of the solution relative to $x \rightarrow-x$ plays the key role to provide extremely small coupling with the radiation continuum in the form of outgoing cylindrical waves for $k_{z}=0$ and $k_{m}=0$ where $k_{m}=2 \pi m / N=0$. The integer $m=0, \pm 1, \pm 2, \ldots, \pm(N-1)$ specifies OAM. For the infinite periodic array of cylinders $k_{m}$ would specify the Bloch wave number along the array.

The radius of the cylinders $a$ is given in terms of the azimuthal period of circular array $h=2 \pi R / N$ where $R$ is the radius of circle. Respectively, the wave numbers are given in terms of inverse $h$. The dispersion curves are computed by solving the dispersion equation $f\left(k_{0}, k_{z}\right)=0$ through analytical continuation of $k_{0}$ into complex plane, where $k_{0}=\omega \mathrm{h} / \mathrm{c}$ is the vacuum wave number, and $k_{z}$ is the propagating constant, the wave number along the cylinders. Fig. 3 shows the real and imaginary parts of complex eigenfrequencies for the case of 20 silicon cylinders. The resonant width and frequency depend on $k_{z}$ quadratically for small $k_{z}$ as seen from Fig. 3. Such a behavior is typical for the guided modes in the vicinity of the $\Gamma$-point in infinite arrays $[26,27,29,34]$. The $Q$ factor of the eigenmode is given by equation $Q=-\operatorname{Re}\left(k_{0}\right) / 2 \operatorname{Im}\left(k_{0}\right)$. Insets in Fig. 3 show profiles of electromagnetic fields (z-components of electric and magnetic field) at $k_{z}=1.5$. This mode converts into a standing wave E-polarized nearly BIC with $H_{z}=0$ at $k_{z}=0$ (see Fig. 5 in Ref. [32]). Therefore when the wave number $k_{z}$ 


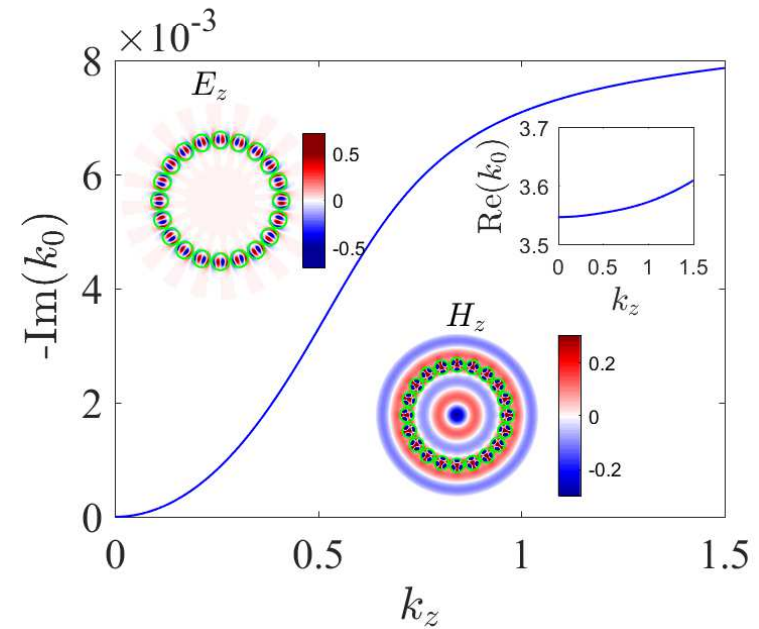

Fig. 3. Leaky zone of resonant modes propagating along the fiber consisted of 20 silicon cylinders with $\epsilon=15$ and radius $a=0.44=0.1382 R$. Insets show z-components of electric and magnetic fields at $k_{0}=3.6086-0.007866 i, k_{z}=1.5, m=0$.

moves away from zero not only the $Q$ factor reduces but also both polarizations are mixed as seen from insets in Fig. 3. One can see that magnetic field fills whole inner space of the fiber as different from the electric field which mostly localized inside the cylinders. That is related to that the electric field is odd relative to $x \rightarrow-x$ to be mostly localized around the cylinders while the magnetic field is even to fill whole inner space of the fiber [32].

The Fig. 4 shows the dispersion curve and the resonant width of the mode which originates from the non-symmetry protected standing E-polarized nearly BIC at $k_{z}=0$. This nearly BIC is symmetry protected in respect to magnetic field and due to tuning the cylinder radius acquires exponentially small coupling with the radiation continuum in respect to the electric field to achieve $Q=2.6 \cdot 10^{8}$. When $k_{z}$ moves away from zero the resonant mode mixes both polarizations. For the infinite array of cylinders electromagnetic field of this nonsymmetry protected BIC were localized around the cylinders. In the circular array the leaky mode has even electric field filling whole core of the fiber, while the odd magnetic field remains localized around the cylinders as shown in insets in Fig. 4.

The most interesting feature of this nearly BIC, however, is a quaternary dependence of the resonant width on $k_{z}$ as shown Fig. 4. Such a behavior of the resonant width was shown in Ref. [28] relative to $k_{z}$ and in Refs. [29,35] relative to the Bloch wave number along the infinite periodic array. Above we considered the leaky zones of the resonant modes in Figs. 3 and 4 originated from standing wave nearly BICs which are suitable for signal transmission along the fiber because of slow velocity of the signal. The Fig. 5 shows the leaky zones which holds BIC point $k_{z c} \neq 0$. One can see the evolution of the resonant width vs the propagation constant with increasing of the cylinder's radius $a$. For the first two choices $a=0.418$ and $a=0.43$ there are two points where the resonant width nearly turns to zero (dash and dash-dot lines in Fig. 5). The first point $k_{z c}=0$ corresponds to the symmetry protected E-polarized standing wave nearly BIC with $Q=1.6 \cdot 10^{7}$ for $a=0.418$ and $Q=5 \cdot 10^{7}$ for $a=0.43$. The second point corresponds to the propagating nearly BIC with mixed polarizations. The propagation constant $k_{z c}$ turns to zero with the increase of cylinder's radius with the

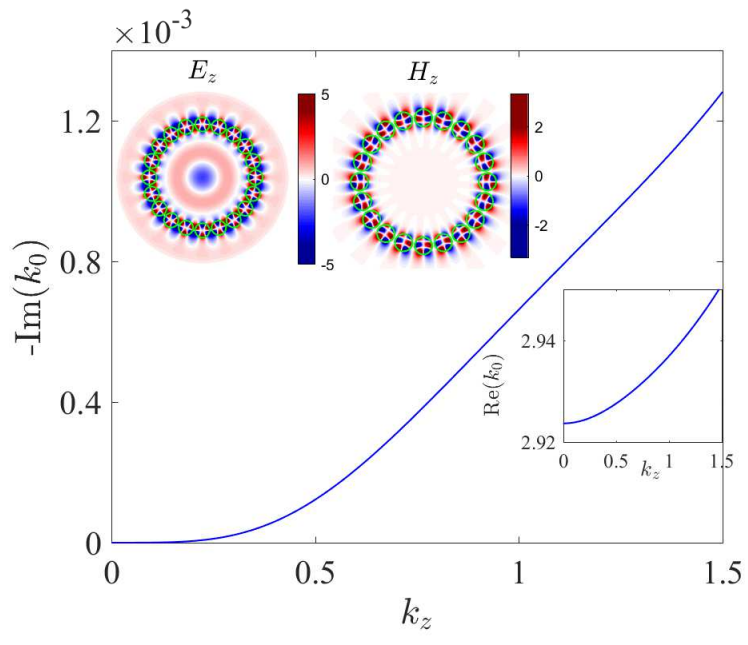

Fig. 4. Leaky zone of resonant mode of the circular array of cylinders with $a=0.43084=0.1354 R$ which converts into the non symmetry protected E-polarized nearly BIC at $k_{z}=0$ and $a=0.43084=0.1354 R$ Insets show $\mathrm{z}$-components of electric and magnetic fields at $k_{0}=2.9508-0.00128 i, m=0, k_{z}=1.5$.

two BICs coalescing at $a=0.453$ at $k_{z c}=0$. The leaky resonant modes hosting this standing wave nearly BIC at the point of coalescence acquires quaternary dependence of the resonant width $-\operatorname{Im}\left(k_{0}\right) \sim k_{z}^{4}$ as shown in Fig. 5 by solid line. That phenomenon was studied in details for the case of the infinite array of cylinders and spheres in Refs. [23, 27] as a result of preservation of topological charge in two-dimensional space of two polarizations of the BIC. It is remarkable for $k_{z}$ in the wide range the resonant width is smaller than $2.5 \cdot 10^{-4}$ as shown in Fig. 5 by dash and dash-dot line. A weak dependence of the $Q$ factor on the wave number allows to use this nearly BIC for signal transmission with high efficiency.

Fig. 6 shows the resonant mode which holds only the propagating nearly BIC at finite values of the wave number but not a standing wave nearly BIC.

This nearly BIC has mixed polarizations with the even electric field $E_{z}$ filling whole core of the fiber and the odd magnetic field localized in the vicinity of cylinders. The mode has $Q$ factor of order $3 \cdot 10^{2}$ at $k_{z}=0$. The $Q$ factor decreases when the wave number goes away from zero but then again goes to extremely large value $3.2 \cdot 10^{8}$ when $k_{z}$ reaches $k_{z}=1.29$ as shown in Fig. 6. The Dispersion curve shows a non-monotonic behavior that is related to an avoided crossing of two neighboring resonances.

The former cases with zero OAM do not need tuning the cylinder radius. Once $m \neq 0$ the propagating wave nearly BICs with nonzero OAM need tuning the radius as it was shown in Ref. [32]. These propagating wave nearly BICs with OAM are shown in Figs. $6(m=1)$ and $7(m=3)$.

\section{DISCUSSION AND CONCLUSIONS}

First, it is interesting to compare propagating nearly BICs in the circular array of cylinders with guided modes propagating along an isolated dielectric cylinder [36]. That comparison is given in Fig. 9. One can see that the frequencies as dependent on the propagation constant $k_{z}$ behave very similar to one another while the resonant widths are strikingly different. If the frequency of the guided mode in the isolated cylinder is be- 


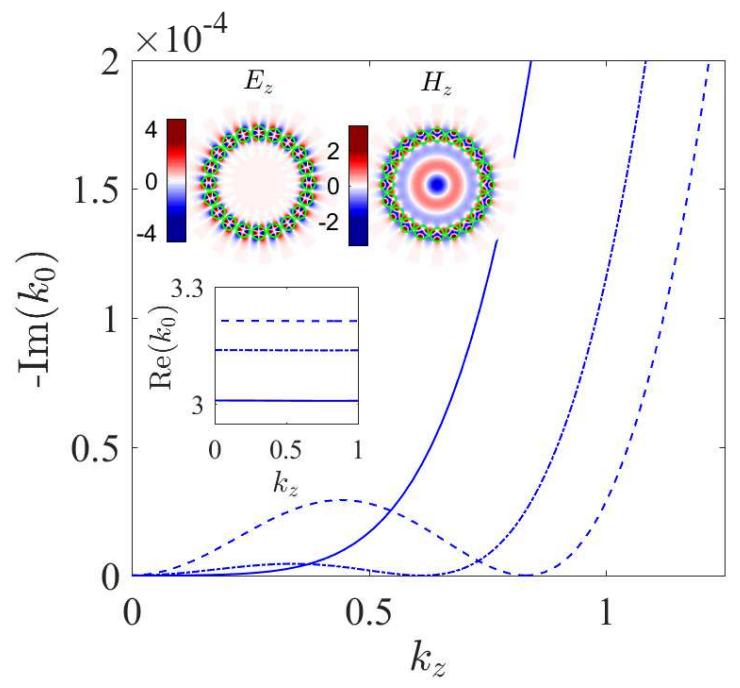

Fig. 5. Leaky zone of resonant mode at $a=0.418=0.1313 R$ (dash lines) with two nearly BICs at $m=0$ and $k_{z}=0$ and $k_{z}=0.83$, at $a=0.43=0.1351 R$ with two nearly BICs at $k_{z}=0$ and $k_{z}=0.605$ which finally collapses into the mode with single nearly BIC at $k_{z}=0$ whose resonant width has an asymptote $k_{z}^{4}$ at $a=0.453=0.1382 R$ (solid lines). Insets show z-components of electric and magnetic fields at $k_{0}=$ $3.2124-3.95 \cdot 10^{-8} i, k_{z}=0.83$ for $a=0.418$.

low the line of light the mode can propagate along the cylinder without leakage. As soon as the frequency is above the line the mode becomes leaky as shown in Fig. 9 (b) by solid line while the widths of the nearly BICs including the resonant modes surrounded the BIC have extremely small leakage above the light line in a rather wide domain of the propagation constant.

Apparently, the choice of the fiber of $N$ dielectric cylinders of circular cross-section is not the best with the technology view point. In general there can be any circular dielectric structure which possess a symmetry relative to azimuthal discrete rotations $\phi \rightarrow \phi+2 \pi n / N$ where $n=1,2,3, \ldots, N$ and $N$ is an integer. In practice the fiber can be chosen in the form of single dielectric cylinder with periodical grating on its surface. The present type fiber composed of $N$ dielectric cylinders has a unique property to exponentially enlarge the $Q$ factor with $N$ $[31,32]$ for specific solutions, i.e., that is nearly BICs. These solutions are localized within the fiber in spite that the frequency of the solution is embedded into the radiation continuum. The fiber can support various nearly BICs mostly standing waves. These BICs are surrounded by weakly leaking resonant modes with the $Q$ factor proportional to inverse of $k_{z}^{2}$. The symmetry protected nearly BICs do not need tuning of the cylinder radius which makes them interesting with the technological point of view. There are also non-symmetry protected nearly BICs which occur via tuning the cylinder radius. The resonant modes surrounding these nearly BICs have extremely weak quaternion dependence of the resonant width on the propagation constant to be specially interesting for signal transmission. The non-symmetry protected propagating nearly BICs surrounded by resonant modes with $Q$ factor inversely proportional to $\left(k_{z}-k_{z c}\right)^{2}$ are the most interesting for signal processing in the fiber. They do not need tuning cylinder radius in contrast to nearly BICs which carry OAM.

It is clear that transmission of electromagnetic signals over

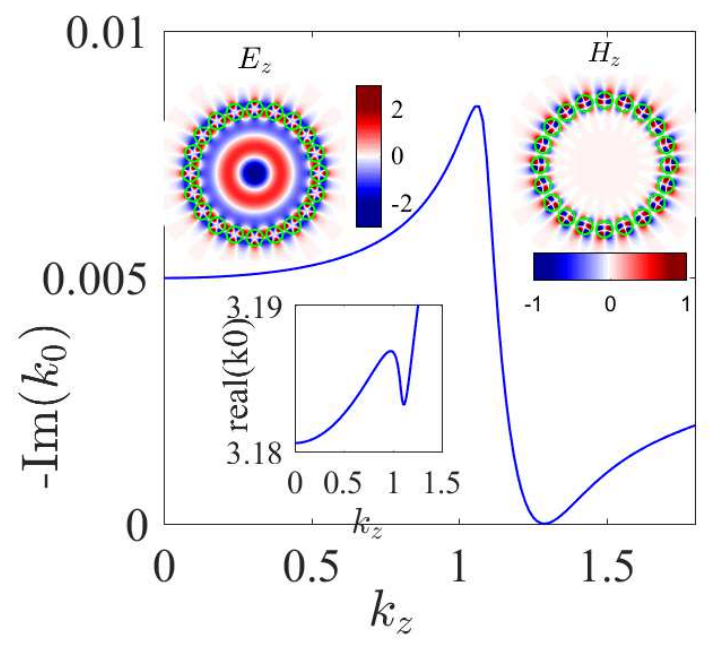

Fig. 6. Leaky zone of resonant mode which converts into the symmetry protected nearly BIC for $a=0.4=0.1257 R$. Insets show z-components of electric and magnetic fields at the complex eigenvalue $k_{0}=3.1916-5 \cdot 10^{-9} i$ and the wave number $k_{z}=1.29, m=0$.

the fiber requires some finite range of frequencies. Because of discreteness of the BIC frequency propagation of signals will be accompanied by leakage. However the majority of resonant widths do not exceed one percent of the frequency. The propagation length which is given by the decay rate of nearly BICs into the radiation continuum. Its value can be accessed as [22]

$$
\frac{L}{\lambda}=\left|\frac{d \operatorname{Re}\left(k_{0}\right)}{d k_{z}}\right| \frac{k_{0}}{2 \pi \operatorname{Im}\left(k_{0}\right)} .
$$

For example we obtain $L / \lambda \approx 10^{4}$ for $a=0.418$ (see Fig. 5 ) and $L / \lambda \approx 5 \cdot 10^{6}$ for $a=0.4$ (see Fig. 6 ) where $\lambda$ is the wavelength. Therefore the propagating nearly BICs can serve for propagation of electromagnetic signals with high quality. That prompts to use the circular array of cylinders as a novel type of optical fibers.

\section{FUNDING INFORMATION}

This work was partially supported by Ministry of Education and Science of Russian Federation (State contract N 3.1845.2017) and the RFBR Grants No.16-02-00314 and No.17-52-45072.

\section{ACKNOWLEDGMENTS}

The authors thank D.N. Maksimov for assistance and discussions. 


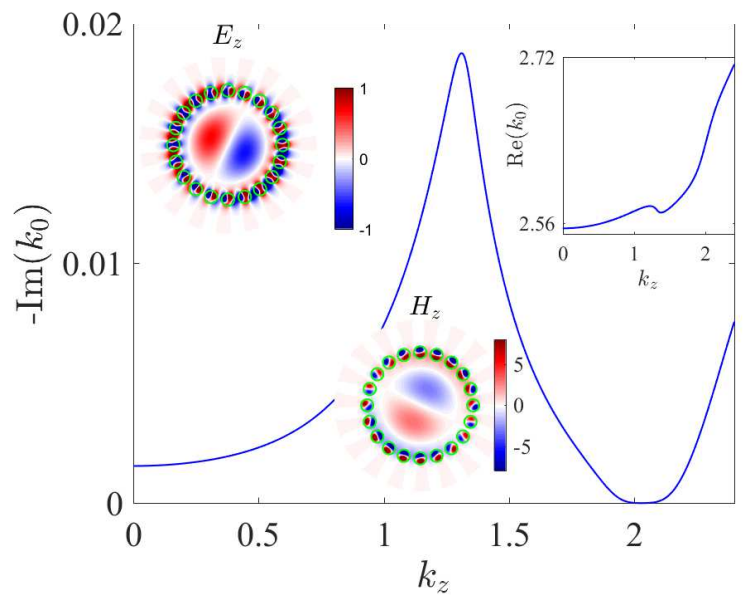

Fig. 7. Resonant mode which converts into the nearly BIC with OAM $m=1$ for $a=0.369=0.116 R$. Insets show zcomponent of the electric field of the propagating nearly BIC at $k_{0}=2.644-5 \cdot 10^{-9} i, k_{z}=2.027$.

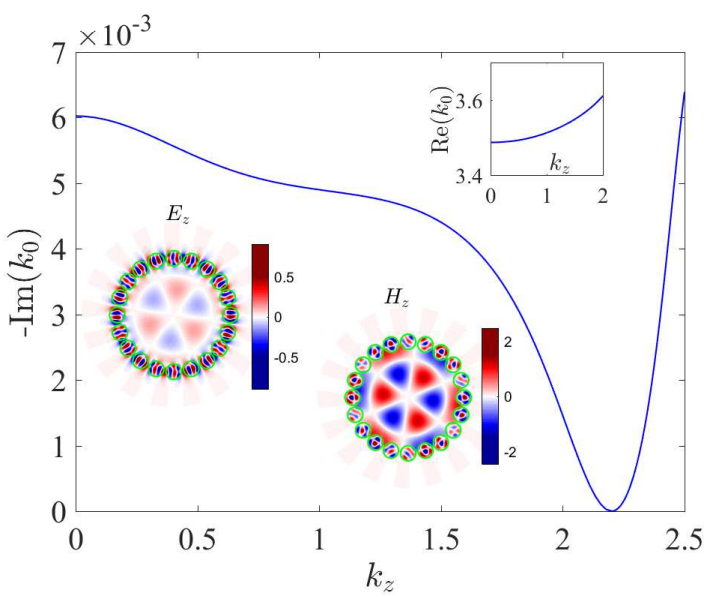

Fig. 8. Leaky zone of resonant mode which converts into the nearly BIC with OAM $m=3$ for $a=0.4327=0.136 R$. Insets show $\mathrm{z}$-component of electric and magnetic fields of the propagating nearly BIC at $k_{0}=3.6544-2 \cdot 10^{-7} i, k_{z}=2.2$.

\section{REFERENCES}

1. J. D. Joannopoulos, R. D. Meade, J. N. Winn, Photonic Crystals: Molding the Flow of Light, (Princeton Univ. Press, Princeton, NJ, 1995).

2. J. C. Knight, J. Broeng, T. A. Birks, P. St. J. Russell, "Photonic Band Gap Guidance in Optical Fibers," Science 285, 1537-1539 (1999).

3. J. C. Knight, T. A. Birks, P. St. J. Russell and D. M. Atkin, "All-silica single-mode optical fiber with photonic crystal cladding," Opt. Lett. 21, 1547-1549 (1996).

4. S.P. Shipman and S. Venakides, "Resonant transmission near non robust periodic slab modes", Phys. Rev. E71, 026611 (2005).

5. D. C. Marinica, A. G. Borisov, and S.V. Shabanov, "Bound States in the Continuum in Photonics", Phys. Rev. Lett. 100, 183902 (2008).

6. Chia Wei Hsu, Bo Zhen, J. Lee, Song-Liang Chua, S.G. Johnson, J.D. Joannopoulos, and M. Soljačić, "Observation
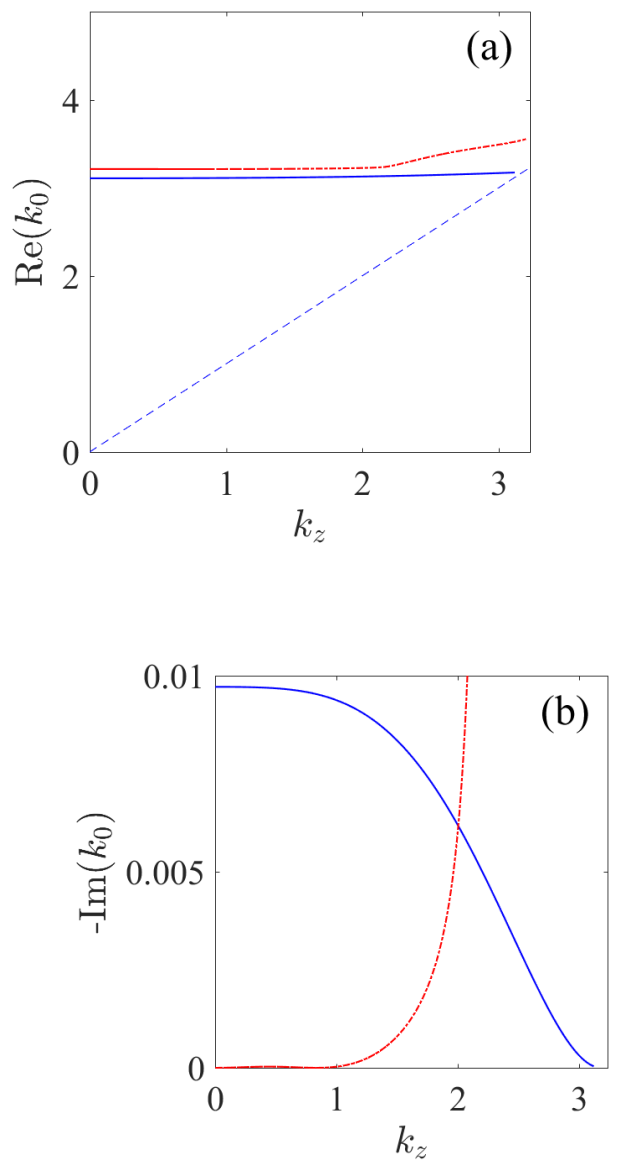

Fig. 9. Dispersion (a) and resonant width (b) of guided mode in isolated dielectric cylinder with $a=0.418$ and $m=0$ (solid lines) compared to the case shown in Fig. 5 (red dashdot lines). Thin dash line shows light line.

of trapped light within the radiation continuum, Nature, 499, 188 (2013).

7. S. Weimann, Yi Xu, R. Keil, A.E. Miroshnichenko, A. Tunnermann, S. Nolte, A.A. Sukhorukov, A. Szameit, and Yu.S. Kivshar, "Compact Surface Fano States Embedded in the Continuum of Waveguide Arrays", Phys. Rev. Lett. 111, 240403 (2013).

8. Chia Wei Hsu, Bo Zhen, Song-Liang Chua, S.G. Johnson, J.D.Joannopoulos, and M. Soljačić, "Bloch surface eigen states with in the radiation continuum", Light: Science and Applications 2, 1 (2013).

9. Bo Zhen, Chia Wei Hsu, Ling Lu, A.D. Stone, and M. Soljačić, "Topological Nature of Optical Bound States in the Continuum", Phys. Rev. Lett. 113, 257401 (2014).

10. Yi Yang, Chao Peng, Yong Liang, Zhengbin Li, and S. Noda, "Analytical Perspective for Bound States in the Continuum in Photonic Crystal Slabs", Phys. Rev. Lett. 113, 037401 (2014).

11. J.M. Foley, S.M. Young, and J.D. Phillips, "Symmetryprotected mode coupling near normal incidence for narrow-band transmission filtering in a dielectric grating", Phys. Rev. B 89, 165111 (2014).

12. Zhen $\mathrm{Hu}$ and $\mathrm{Ya}$ Yan $\mathrm{Lu}$, "Standing waves on two- 
dimensional periodic dielectric waveguides", J. Optics, 17, 065601 (2015).

13. Maowen Song, Honglin $\mathrm{Yu}$, Changtao Wang, Na Yao, Mingbo Pu, Jun Luo, Zuojun Zhang, and Xiangang Luo, "Sharp Fano resonance induced by a single layer of nanorods with perturbed periodicity", Opt. Express, 23, 2895 (2015).

14. Lijun Yuan and Ya Yan Lu, "Diffraction of plane waves by a periodic array of nonlinear circular cylinders," Phys. Rev. A 94, 013852 (2016).

15. Zhixin Wang, Hanxing Zhang, Liangfu Ni, Weiwei $\mathrm{Hu}$, and Chao Peng, "Analytical Perspective of Interfering Resonances in High-Index-Contrast Periodic Photonic Structures", IEEE J. Quant. Electr. 52, 6100109 (2016).

16. Z.F. Sadrieva, I.S Sinev, K.L. Koshelev, A. Samusev, I.V. Iorsh, O. Takayama, R. Malureanu, A.A. Bogdanov, and A.V. Lavrinenko, "Transition from optical bound states in the continuum to leaky resonances: role of substrate and roughness," ACS Photonics 4, 723 (2017).

17. E.N. Bulgakov and A.F. Sadreev, "Trapping of light with angular orbital momentum above the light cone in a periodic array of dielectric spheres", Adv. EM, 6, 1 (2017).

18. Lijun Yuan and Ya Yan Lu, "Bound states in the continuum on periodic structures: perturbation theory and robustness", Opt. Lett. 42, 4490-4493 (2017).

19. Zhen Hu and Ya Yan Lu, "Resonances and Bound States in the Continuum on Periodic Arrays of Slightly Noncircular Cylinders", J. Phys. B: At. Mol. Opt. Phys. (2017).

20. E.N. Bulgakov and A.F. Sadreev, "Bloch bound states in the radiation continuum in a periodic array of dielectric rods", Phys. Rev. A90, 053801 (2014).

21. Chang-Ling Zou, Jin-Ming Cui, Fang-Wen Sun, Xiao Xiong, Xu-Bo Zou, Zheng-Fu Han, and Guang-Can Guo, "Guiding light through optical bound states in the continuum for ultrahigh-Q microresonators", Laser Photonics Rev. 9, 114 (2015).

22. E.N. Bulgakov and D.N. Maksimov, "Light guiding above the light line in arrays of dielectric nanospheres," Opt. Lett. 41, 3888-3891 (2016).

23. Lijun Yuan and Ya Yan Lu, "Propagating Bloch modes above the lightline on a periodic array of cylinders", J. Phys. B: At. Mol. Opt. Phys. 50, 05LT01 (2017).

24. Zhen $\mathrm{Hu}$ and Ya Yan Lu, "Propagating bound states in the continuum at the surface of a photonic crystal", J. Opt. Soc. Am. B 34, 1878-1883 (2017).

25. Bo Zhen, Chia Wei Hsu, Ling Lu, A.D. Stone, and M. Soljačić, " Topological Nature of Optical Bound States in the Continuum,", Phys. Rev. Lett. 113, 257401 (2014).

26. Lijun Yuan and Ya Yan Lu, "Strong resonances on periodic arrays of cylinders and optical bistability with weak incident waves", Phys. Rev. A 95, 023834 (2017).

27. E.N. Bulgakov and D.N. Maksimov, "Topological Bound States in the Continuum in Arrays of Dielectric Spheres", Phys. Rev. Lett. 118, 267401 (2017).

28. E.N. Bulgakov and D.N. Maksimov, "Bound states in the continuum and polarization singularities in periodic arrays of dielectric rods", Phys. Rev. A 96, 063833 (2017).

29. E.N. Bulgakov and D.N. Maksimov, "Light enhancement by quasi-bound states in the continuum in dielectric arrays,", Opt. Express 25, 14134-14147 (2017).

30. L. Ni, J. Jin, C. Peng, and Z. Li, "Analytical and statistical investigation on structural fluctuations induced radiation in photonic crystal slabs,", Opt. Express 25, 5580-5593
(2017).

31. Hai-bin Lü and Xiaoping Liu, "Trapped modes with extremely high quality factor in the subwavelength ring resonator composed of dielectric nanorods", arXiv:1709.08006 (2017).

32. E.N. Bulgakov and A.F. Sadreev, "Nearly bound states in the radiation continuum in a circular array of dielectric rods," Phys. Rev. A97, 033834 (2018).

33. D. Maystre, S. Enoch, and G. Tayeb, "Scattering Matrix Method Applied to Photonic Crystals" in book Electromagnetic Theory and Applications for Photonic Crystals ed. by K. Yasumoto (Taylor \& Francis Group, LLC, 2006).

34. I.Ya. Polishchuk, A.A. Anastasiev, E.A. Tsyvkunova, M.I. Gozman, S.V. Solovòv, and Yu. I. Polishchuk, "Guided modes in the plane array of optical waveguid", Ph7ys. Rev. A 95, 053847 (2017).

35. Lijun Yuan and Ya Yan Lu, "Bound states in the continuum on periodic structures surrounded by strong resonances," Phys. Rev. A97, 043828 (2018).

36. J. D. Jackson, Classical Electrodynamics (John Wiley and Sons, Inc., New York, 1962). 